\title{
UTILIZAÇÃO DE SORO DE LEITE E AMIDO NA PRODUÇÃO DE DOCE DE LEITE PASTOSO: RENDIMENTO, COMPOSIÇÃO, PERFIL DE TEXTURA, VISCOSIDADE E AVALIAÇÃO SENSORIAL DE ACEITAÇÃO
}

\section{Whey and starch application for pasty dulce de leche production: yield, composition, texture profile, viscosity, and sensory evaluation of acceptance}

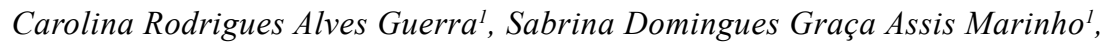
Rodrigo Stephani ${ }^{2}$ Isis Rodrigues Toledo Renhe ${ }^{3}$, Antônio Fernandes de Carvalho ${ }^{1}$, Ítalo Tuler Perrone ${ }^{*}$
\end{abstract}

\section{RESUMO}

O soro pode ser empregado na produção do doce de leite desde que a composição do produto final esteja de acordo com a legislação. Neste estudo, misturas de leite, soro, açúcar e amido foram utilizadas para a produção de doce de leite. De acordo com os resultados, os doces com 15\% de adição de soro e sem adição de soro, ambos sem adição de amido, configuraram as melhores formulações sob o aspecto de aceitação sensorial. Misturas de 30\% de soro com $70 \%$ de leite empregando ou não amido não diferiram estatisticamente entre si segundo a análise de aceitação sensorial, contudo apresentam composição não conforme com a legislação. Nas condições do experimento, valores mais elevados de dureza, gomosidade, adesividade e viscosidade implicaram em notas mais elevadas na análise sensorial. Os efeitos da adição de soro e amido no rendimento e na composição do doce de leite são descritos.

Palavras-chave: concentração; coproduto; reologia.

1 Universidade Federal de Viçosa, Departamento de Tecnologia de Alimentos, Viçosa, MG, Brasil.

2 Universidade Federal de Juiz de Fora, Departamento de Química, Juiz de Fora, MG, Brasil.

3 Empresa de Pesquisa Agropecuária de Minas Gerais, Instituto de Laticínios Cândido Tostes, Juiz de Fora, MG.

4 Universidade Federal de Juiz de Fora, Faculdade de Farmácia, Rua José Lourenço Kelmer, s/n, São Pedro, 36036-900, Juiz de Fora, MG, Brasil. E-mail: italotulerperrone@gmail.com

* Autor para correspondência

Recebido / Received: 10/05/2019

Aprovado / Approved: 17/03/2020 


\begin{abstract}
Whey is allowed as an ingredient for dulce de leche production since the final composition of the product fits the standards of Legislation. In this study, mixtures of milk, whey, sugar, and starch were applied to produce dulce de leche. According to the results, the mixtures of $15 \%$ of whey and without added whey, both without starch addition, presented the best formulations under the aspect of sensory acceptance. Mixtures of $30 \%$ of whey and $70 \%$ of milk using or not starch did not differ significantly among them according to the sensorial panel, but they did not fit the required composition standards. Under the conditions of the experiment, higher values of hardness, gumminess, adhesiveness, and viscosity lead to higher scores in the sensorial panel. The effects of whey and starch addition on yield and composition of dulce de leche are described.
\end{abstract}

Keywords: concentration; co-product; rheology.

\section{INTRODUÇÃO}

Tecnologicamente, o doce de leite se enquadra entre os derivados de leite conservados por evaporação e adição de açúcar e é devido à alta pressão osmótica criada que pode ser conservado em temperatura ambiente. O doce pode apresentar consistência cremosa ou pastosa e deve ser homogêneo, sem grumos ou bolhas, possuir sabor e aroma característicos e uma cor caramelo que é consequente da Reação de Maillard. É permitido o uso de diferentes aditivos e coadjuvantes de processamento, desde que sua composição final se encontre dentro da legislação vigente, em destaque teor de adição de amido de $0,5 \%$ sobre o volume de leite, teores de gordura no doce entre 6 a $9 \%$, teor máximo de cinzas de $2 \%$, teor mínimo de proteína de $5 \%$ e umidade máxima de 30\% (BRASIL, 1997).

Diversos estudos recentes buscam caracterizar o processo de produção e o produto, bem como descrever cientificamente as interações entre formulação e processo nas propriedades do doce de leite (CARNEIRO et al., 2020; FRANCISQUINI et al., 2019; FRANCISQUINI et al., 2018a; FRANCISQUINI et al., 2018b; PAIVA et al., 2018; OLIVEIRA et al., 2017; FRANCISQUINI et al., 2016; SILVA et al., 2015).
Segundo Perrone et al. (2006), a adição de soro de leite às matérias-primas leite e açúcar em formulações de doce de leite pode possibilitar a obtenção de produto semelhante ao tradicional em composição e atributos sensoriais.

De acordo com Silva et al. (2015) a adição de amido na dosagem de $0,5 \%$ sobre o volume de leite contribui para o controle da cristalização da lactose durante o armazenamento do doce de leite, contudo nesta dosagem não contribui significativamente para o rendimento da produção.

Francisquini et al. (2016), caracterizaram o perfil de textura de doces de leite industriais tendo encontrado correlações significativas entre o comportamento reológico dos produtos e os valores de atividade de água e de umidade, independentemente da rota tecnológica empregada durante a produção dos mesmos. A análise do perfil da textura, também conhecido como TPA, é feita em um aparelho constituído por um dinamômetro que fornece energia mecânica a velocidade constante. Fornece a curva de força versus tempo, onde é registrado, de acordo com a geometria usada no teste, a variação da textura do material. A maior vantagem da avaliação do TPA é que com este pode-se aferir muitas outras variáveis no segundo ciclo 
de compressão, como: dureza, elasticidade, coesividade, adesividade, fraturabilidade, resiliência, gomosidade, mastigabilidade, entre outras (HUIDOBRO et al., 2005).

O objetivo deste trabalho foi avaliar o efeito da utilização do soro de leite e amido nos atributos de rendimento, composição, reologia e na avaliação sensorial de doce de leite pastoso.

\section{MATERIAL E MÉTODOS}

Os trabalhos experimentais foram realizados no Laboratório de Novos Produtos, Departamento de Tecnologia de Alimentos (DTA) da Universidade Federal de Viçosa (UFV), em Viçosa, MG. As formulações empregadas no experimento são apresentadas na Tabela 1.

As produções de doce de leite foram realizadas com três repetições e a composição média do leite empregado nas fabricações foi: $4,6 \pm 0,1 \% \mathrm{~m} / \mathrm{m}$ de lactose, $3,2 \pm 0,1 \% \mathrm{~m} / \mathrm{m}$ de proteínas, $3,2 \pm 0,2 \% \mathrm{~m} / \mathrm{m}$ de gordura e $0,8 \pm 0,1 \% \mathrm{~m} / \mathrm{m}$ de cinzas. A composição média dos soros empregados no experimento foi: $4,7 \pm 0,1 \% \mathrm{~m} / \mathrm{m}$ de lactose, $1,0 \pm 0,1 \% \mathrm{~m} / \mathrm{m}$ de proteínas, $0,3 \pm 0,1 \% \mathrm{~m} / \mathrm{m}$ de gordura e $0,8 \pm 0,0 \% \mathrm{~m} / \mathrm{m}$ de cinzas.
Os doces foram produzidos segundo Perrone et al. (2011). O ponto do doce foi padronizado para teor de sólidos solúveis de $69 \pm 1{ }^{\circ}$ Brix. O amido empregado nas fabricações foi o amido de milho modificado por esterificação cedido pela empresa Tate \& Lyle Gemacom Tech e adicionado quando o teor de sólidos solúveis do doce era de $46 \pm 1{ }^{\circ}$ Brix.

Os produtos obtidos foram analisados quanto aos teores de cinzas (incineração em mufla), proteínas (método Kjeldahl) e gordura (Método Gerber) segundo as metodologias oficiais definidas pelo Ministério da Agricultura, Pecuária e Abastecimento MAPA (BRASIL, 2018).

O teor de adição de bicarbonato de sódio utilizado em todos os tratamentos foi de $0,1 \%$ $\mathrm{m} / \mathrm{v}$ objetivando padronizar as formulações empregadas, fazendo com que as variáveis do experimento fossem o teor de adição de soro e a adição de amido.

As análises de rendimento foram realizadas por meio de balanço de massa dos ingredientes usados para fabricação do doce de leite e a quantidade de doce final produzida de acordo com Perrone et al. (2012). O percentual de rendimento foi obtido pela razão entre a massa de doce obtida no experimento e o valor calculado (Equação 1).

Tabela 1 - Formulações empregadas no experimento $(n=3)$

\begin{tabular}{cccc}
\hline $\begin{array}{c}\text { Percentual de soro na mistura* } \\
\text { leite e soro destinada à produção do } \\
\text { doce de leite }\end{array}$ & $\begin{array}{c}\text { Sacarose } \\
(\% \mathrm{~m} / \mathrm{v})\end{array}$ & $\begin{array}{c}\text { Bicarbonato de sódio } \\
(\% \mathrm{~m} / \mathrm{v})\end{array}$ & $\begin{array}{c}\text { Amido } \\
(\% \mathrm{~m} / \mathrm{v})\end{array}$ \\
\hline 0 & 20 & 0,1 & 0,0 \\
0 & 20 & 0,1 & 0,5 \\
15 & 20 & 0,1 & 0,0 \\
30 & 20 & 0,1 & 0,5 \\
30 & 20 & 0,1 & 0,0 \\
\hline
\end{tabular}

*A massa final de mistura em cada produção foi de $12 \mathrm{~kg}$. 
O tempo de evaporação foi medido por cronômetro, desde o início do processamento no tacho encamisado até a evaporação desejada da água e obtenção do teor de sólidos desejado.

A análise sensorial de aceitação foi realizada no Laboratório de Análise Sensorial no Edifício Beck Andersen (EBA) da Universidade Federal de Viçosa, MG com 112 provadores não treinados (55 homens e 57 mulheres, com idade entre 16 e 37 anos), que analisaram as amostras numa escala hedônica de 1 a 9 , onde o limite mínimo da escala foi a descrição "Desgostei extremamente", equivalente a pontuação 1 e o limite máximo a descrição "Gostei extremamente", equivalente a pontuação 9. As amostras foram apresentadas de forma monádica em pequenos copos brancos enumerados com algarismos de três dígitos, seguindo-se delineamento de blocos completos casualizados. Os resultados foram analisados por meio da Análise de Variância (teste F) a 5\% de significância.

O perfil de textura (TPA) dos doces foi avaliado em duplicata, empregando-se um Texturômetro Brookfield CT3. Com isso, obtiveram-se os valores dos seguintes atributos descritos por Szczesniak (1963): dureza - força necessária para produzir uma deformação na amostra; adesividade - energia necessária para superar as forças atrativas entre a superfície do alimento e de outros materiais com as quais o alimento está em contato; gomosidade - energia requerida para desintegrar um alimento até estar pronto para a deglutição.

As amostras foram armazenadas em potes plásticos de forma cônica (com diâmetro maior de 9,2 cm, diâmetro menor de 7,0 cm e altura de $8,1 \mathrm{~cm}$ ) à temperatura ambiente, e foram avaliadas no recipiente em que estavam guardadas. Para o teste de textura foram adotados os seguintes parâmetros: velocidade de pré teste $-2 \mathrm{~mm} / \mathrm{s}$; velocidade teste - $1 \mathrm{~mm} / \mathrm{s}$; distância - $20,0 \mathrm{~mm}$; tempo - 0,0 s; força de contato - 50,0 gf; ponta de prova - TA25/1000.

A viscosidade foi determinada a $25 \pm 2{ }^{\circ} \mathrm{C}$ em Viscosímetro Analógico Brookfield LVT empregando probe número 1 com rotação de $60 \mathrm{rpm}$ e o resultado foi expresso em mPa.s.

\section{RESULTADOS E DISCUSSÃO}

$\mathrm{Na}$ Tabela 2, são apresentados os resultados das análises de composição dos doces.

Verifica-se a partir dos resultados apresentados na Tabela 2 que todos os doces produzidos no experimento atenderam aos

\section{Equação 1}

$$
\mathrm{MDL}=\frac{[(\mathrm{MLI} \times \% \mathrm{SLLI})+(\mathrm{MS} \times \% \mathrm{SLS})+(\mathrm{MSC}+\mathrm{MR}+\mathrm{ME})]}{\% \mathrm{STDL}}
$$

Sendo:

$\mathrm{MDL}=$ quantidade de doce final (m)

$\mathrm{MLI}=$ massa de leite integral $(\mathrm{kg})$ ou volume de leite $(\mathrm{L})$;

$\%$ SLLI $=$ porcentagem de sólidos láticos do leite integral $(\% \mathrm{~m} / \mathrm{m}$ ou $\% \mathrm{~m} / \mathrm{v})$;

$\mathrm{MS}=$ massa de soro $(\mathrm{kg})$ ou volume de soro $(\mathrm{L})$;

$\% \mathrm{SLS}=$ porcentagem de sólidos láticos do soro $(\% \mathrm{~m} / \mathrm{m}$ ou $\% \mathrm{~m} / \mathrm{v})$;

$\mathrm{MSC}=$ massa se sacarose $(\mathrm{kg})$;

$\mathrm{MR}=$ massa de redutor de acidez bicarbonato de sódio $(\mathrm{kg})$;

$\mathrm{ME}=$ massa de espessante $(\mathrm{kg})$;

$\% \mathrm{STDL}=$ porcentagem de sólidos totais do doce de leite $(\% \mathrm{~m} / \mathrm{m})$ 
atributos teores de umidade, proteínas e cinzas estipulado pela legislação.

$\mathrm{O}$ atributo teor de gordura é um limitante à adição de soro ao leite para produção de doce de leite em que se utilizou concentração de $30 \%$ de soro, tanto na mistura com amido como naquela sem amido, em que os teores de gordura ficaram abaixo do estipulado pela legislação.

Os resultados de massa obtida de doce de leite final $(\mathrm{kg})$ e do rendimento expresso em massa de mistura inicial por massa de produto $(\mathrm{kg} / \mathrm{kg})$ são apresentados na Tabela 3.

Depreende-se dos resultados de rendimento que a adição de $0,5 \%$ de amido não implicou no aumento do rendimento, resultado semelhante foi encontrado por Silva et al. (2015).

A adição de soro diminui o tempo de fabricação de acordo com os dados apresentados na Tabela 3 relativos ao doce sem amido, assim como na produção com participação de $30 \%$

Tabela 2 - Valores da composição centesimal das amostras de doce de leite e suas respectivas exigências legais $(n=3 *)$

\begin{tabular}{cccccc}
\hline \multirow{2}{*}{ Doce de Leite } & $\begin{array}{c}\text { Percentual de soro na } \\
\text { mistura leite e soro }\end{array}$ & Umidade (\%) & Gordura (\%) & Cinzas (\%) & Proteína (\%) \\
\hline \multirow{3}{*}{ Adicionado de amido } & 0 & $27,80 \pm 2,01$ & $7,0 \pm 0,5$ & $1,68 \pm 0,16$ & $7,55 \pm 0,15$ \\
& 15 & $25,93 \pm 1,94$ & $6,0 \pm 0,0$ & $1,65 \pm 0,10$ & $6,43 \pm 0,17$ \\
& 30 & $25,47 \pm 2,03$ & $5,0 \pm 0,5$ & $1,67 \pm 0,10$ & $6,01 \pm 0,09$ \\
\hline \multirow{2}{*}{ Sem adição de amido } & 0 & $26,20 \pm 2,05$ & $8,0 \pm 0,5$ & $1,92 \pm 0,08$ & $7,55 \pm 0,10$ \\
& 15 & $25,23 \pm 1,57$ & $6,0 \pm 0,5$ & $1,74 \pm 0,09$ & $6,43 \pm 0,12$ \\
\hline $\begin{array}{c}\text { Portaria no 354/1997 } \\
\text { MAPA }\end{array}$ & 30 & $25,63 \pm 2,63$ & $4,0 \pm 0,0$ & $1,65 \pm 0,07$ & $5,57 \pm 0,11$ \\
\hline
\end{tabular}

*A formulação sem soro foi fabricada sem repetição.

Tabela 3 - Tempos de evaporação e valores das massas esperada e real de doce de leite, e dos respectivos rendimentos das formulações com 0,15 e $30 \%$ de soro adicionado ao leite, nos processos com e sem amido*

\begin{tabular}{cccccc}
\hline $\begin{array}{c}\text { Doce de } \\
\text { Leite }\end{array}$ & $\begin{array}{c}\text { Percentual de soro } \\
\text { na mistura leite e } \\
\text { soro }\end{array}$ & $\begin{array}{c}\text { Massa } \\
\text { calculada de } \\
\text { doce }(\mathrm{kg})^{1}\end{array}$ & $\begin{array}{c}\text { Massa obtida } \\
\text { de doce }(\mathrm{kg})\end{array}$ & $\begin{array}{c}\text { Rendimento } \\
(\mathrm{kg} / \mathrm{kg})\end{array}$ & $\begin{array}{c}\text { Tempo de } \\
\text { evaporação (min) }\end{array}$ \\
\hline \multirow{3}{*}{ Com amido } & 0 & 5,5 & $4,9^{\mathrm{a}}$ & $2,1^{\mathrm{a}}$ & $84,0^{\mathrm{a}} \pm 3,5$ \\
& 15 & 5,2 & $5,1^{\mathrm{a}}$ & $1,9^{\mathrm{a}}$ & $89,5^{\mathrm{a}} \pm 4,8$ \\
& 30 & 5,0 & $5,0^{\mathrm{a}}$ & $1,7^{\mathrm{a}}$ & $79,0^{\mathrm{b}} \pm 2,7$ \\
\hline \multirow{3}{*}{ Sem amido } & 0 & 5,3 & $4,7^{\mathrm{a}}$ & $2,1^{\mathrm{a}}$ & $84,0^{\mathrm{a}} \pm 4,1$ \\
& 15 & 5,1 & $5,1^{\mathrm{a}}$ & $1,9^{\mathrm{a}}$ & $75,0^{\mathrm{b}} \pm 3,1$ \\
\hline
\end{tabular}

1 massa calculada é igual ao rendimento teórico calculado pela equação 1 .

* Médias seguidas pela mesma letra não diferem estatisticamente ao nível de $5 \%$ de probabilidade teste de Tukey. 
de soro na mistura e adicionado de amido. Teores mais elevados de soro na mistura leite e soro fazem com que maior quantidade de água tenha que ser evaporada. Desta forma, a redução no volume de mistura dentro do tacho é maior. Observou-se que durante o processo a maior redução no volume propiciou que a mesma área de troca de energia na forma de calor (área da parede do tacho) intensificou a evaporação. Nas condições do experimento a maior adição de soro na mistura levou a maior quantidade de água a ser evaporada, contudo a maior redução no volume nestes produtos propiciou evaporação mais rápida devido a relação entre o volume de produto e a área da superfície trocadora de calor. Essa redução do tempo de preparo implica em redução de custos para empresa, principalmente com energia e possibilidade de aumento de produção. $\mathrm{O}$ mesmo comportamento foi obtido no trabalho de Silva et al. (2013).

A partir das fichas preenchidas na análise sensorial, a média obtida por cada formulação é observada na Tabela 4. Observase que houve diferença entre as amostras quanto à aceitação $(\mathrm{p}<0,05)$.

Tabela 4 - Resultados médios da análise sensorial (teste de aceitação) de doce de leite fabricado com diferentes percentuais de soro de leite, com e sem adição de amido

\begin{tabular}{cc}
\hline $\begin{array}{c}\text { Tratamento do Doce de } \\
\text { Leite }\end{array}$ & Nota média* $^{*}$ \\
\hline $15 \%$ sem amido & $7,73^{\mathrm{a}}$ \\
$0 \%$ sem amido & $7,33^{\mathrm{ab}}$ \\
$15 \%$ com amido & $7,1^{\mathrm{b}}$ \\
$0 \%$ com amido & $7,04^{\mathrm{b}}$ \\
$30 \%$ sem amido & $7,03^{\mathrm{b}}$ \\
$30 \%$ com amido & $6,97^{\mathrm{b}}$
\end{tabular}

* Médias seguidas pela mesma letra não diferem estatisticamente ao nível de $5 \%$ de probabilidade teste de Tukey.
De acordo com os resultados apresentados na Tabela 4, os doces que atingiram os dois maiores escores na análise sensorial foram, respectivamente, os doces com $15 \%$ de adição de soro e sem adição de soro, ambos sem adição de amido. Deve-se ressaltar que a adição de $15 \%$ de soro ao leite não difere estatisticamente da tecnologia sem soro (ambas sem adição de amido), desta forma a adição de $15 \%$ de soro não impacta negativamente na aceitação do doce de leite sem amido. A obtenção de resultados favoráveis à produção de doce de leite adicionado de soro é descrita em Perrone et al. (2008). Excluindo a formulação de $15 \%$ de soro sem amido, as demais não diferiram entre si ( $p>0,05)$, portanto não houve variação na aceitação dos doces para esses níveis de adição de soro, com ou sema adição de amido.

O resultado da análise reológica das diferentes fabricações de doce de leite está presente nas Figuras 1 e 2, para as formulações com e sem amido, respectivamente.

A interpretação dos gráficos acima fornece os valores expostos na Tabela 5, com as médias obtidas na análise sensorial.

Observa-se para os doces sem amido que os valores de viscosidade diminuem de 30.800 mPa.s para $22.133 \mathrm{mPa}$.s ao se passar de uma formulação sem soro para uma com $15 \%$. E ao se trabalhar com $30 \%$ de soro na formulação observa-se redução da viscosidade para $16.533 \mathrm{mPa}$.s. A adição progressiva de soro ao leite para a produção do doce de leite acarreta diminuição significativa da viscosidade, o que pode ser atribuído à mudança na proporção de caseína e soro proteína causada pela adição do soro. A amplitude da viscosidade foi de $14.267 \mathrm{mPa}$.s quando se compara o doce obtido somente a partir do leite e obtido a partir da mistura de $30 \%$ de soro com $70 \%$ de leite. A mesma amplitude para estes tratamentos quando empregou-se o amido foi de apenas $4.933 \mathrm{mPa} . \mathrm{s}$ (2,89 vezes menor) e não observou-se diferença significativa na viscosidade quando o teor de soro passou de 


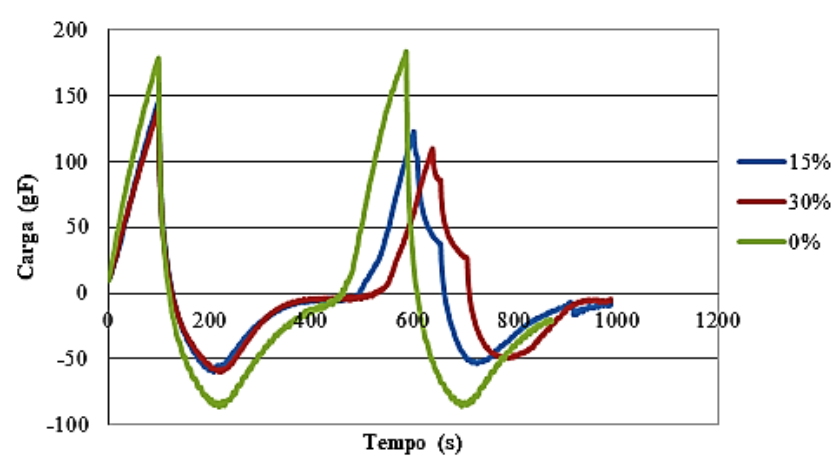

Figura 1 - Análise de perfil de textura para as diferentes formulações, com adição de amido

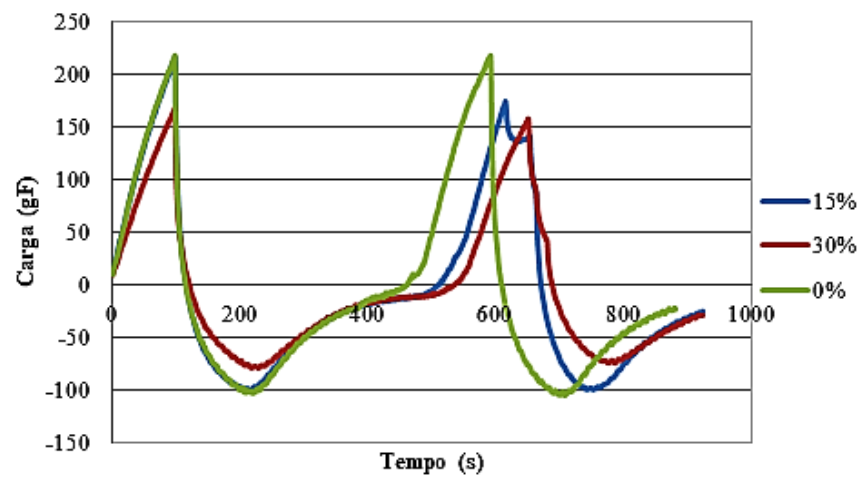

Figura 2 - Análise de perfil de textura para as diferentes formulações, sem adição de amido

Tabela 5 - Resultados de perfil de textura, viscosidade, teor de umidade e análise de aceitação sensorial em doces de leite fabricados com diferentes concentrações de soro, com e sem adição de amido*

\begin{tabular}{cccccccc}
\hline $\begin{array}{c}\text { Doce de } \\
\text { Leite }\end{array}$ & $\begin{array}{c}\text { Percentual } \\
\text { de soro na } \\
\text { mistura leite } \\
\text { e soro }\end{array}$ & $\begin{array}{c}\text { Dureza 1 } \\
\text { (gf) }\end{array}$ & $\begin{array}{c}\text { Dureza } \\
\text { (gf) }\end{array}$ & $\begin{array}{c}\text { Gomosidade } \\
\text { (gf) }\end{array}$ & $\begin{array}{c}\text { Adesividade } \\
(\mathrm{mJ})\end{array}$ & $\begin{array}{c}\text { Viscosidade } \\
(\mathrm{mPa} . \mathrm{s})\end{array}$ & $\begin{array}{c}\text { Teor de } \\
\text { umidade }\end{array}$ \\
\hline Com & 0 & $178,0^{\mathrm{b}}$ & $183,0^{\mathrm{b}}$ & $186,2^{\mathrm{b}}$ & $13,6^{\mathrm{b}}$ & $20.000^{\mathrm{b}}$ & $27,80^{\mathrm{a}}$ \\
amido & 15 & $145,5^{\mathrm{c}}$ & $147,8^{\mathrm{c}}$ & $142,7^{\mathrm{c}}$ & $8,2^{\mathrm{c}}$ & $16.400^{\mathrm{c}}$ & $25,93^{\mathrm{b}}$ \\
& 30 & $140,5^{\mathrm{c}}$ & $144,2^{\mathrm{c}}$ & $147,5^{\mathrm{c}}$ & $8,2^{\mathrm{c}}$ & $15.067^{\mathrm{c}}$ & $25,47^{\mathrm{c}}$ \\
\hline \multirow{2}{*}{ Sem } & 0 & $219,0^{\mathrm{a}}$ & $219,0^{\mathrm{a}}$ & $227,1^{\mathrm{a}}$ & $16,5^{\mathrm{a}}$ & $30.800^{\mathrm{a}}$ & $26,20^{\mathrm{b}}$ \\
amido & 15 & $217,0^{\mathrm{a}}$ & $219,5^{\mathrm{a}}$ & $215,4^{\mathrm{a}}$ & $16,8^{\mathrm{a}}$ & $22.133^{\mathrm{b}}$ & $25,23^{\mathrm{c}}$ \\
\hline & 30 & $168,5^{\mathrm{bc}}$ & $169,8^{\mathrm{bc}}$ & $164,5^{\mathrm{bc}}$ & $13,6^{\mathrm{b}}$ & $16.533^{\mathrm{c}}$ & $25,63^{\mathrm{c}}$ \\
\hline
\end{tabular}

*Médias seguidas pela mesma letra (na coluna) não diferem estatisticamente ao nível de $5 \%$ de probabilidade teste de Tukey. 
$15 \%$ para $30 \%$ na presença de amido. $\mathrm{O}$ amido possui papel importante na viscosidade do doce de leite, diminuindo a faixa de variação desta propriedade quando adiciona-se soro na formulação.

A análise instrumental do perfil de textura mostrou uma tendência de que os doces apresentaram valores menores de dureza, adesividade, gomosidade e elasticidade quando foram fabricados com maiores teores de soro. Pode-se perceber que os maiores escores na análise sensorial foram as formulações que obtiveram valores maiores de dureza 1 e 2 , gomosidade e adesividade. Os valores de dureza expressam a força para deformar a amostra, a força para desintegrar o doce, o que é mais aceitável pelo consumidor do que um doce com pequena dureza. A gomosidade já se relaciona à energia necessária para desintegrar o doce antes do mesmo ser deglutido. Também houve uma maior aceitação em doces para os quais essa energia requerida é maior. Maiores valores para adesividade proporcionaram maior aceitação sensorial. As formulações que permaneceram mais tempo aderidas à boca foram as mais aceitas quando comparadas às de menor adesividade. O mesmo foi observado quanto à viscosidade do produto final.

\section{CONCLUSÃO}

A adição de soro ao leite para a produção de doce de leite permite a obtenção de produtos com boa aceitação sensorial, contudo em relação aos teores de adição deve-se observar a mudança na composição final que acarretam, em especial ao teor de gordura. A adição do amido não alterou a composição físico química do produto e nem o rendimento quando mantido constante os percentuais de leite e soro na mistura. De acordo com as condições deste experimento a maior aceitação do doce de leite está ligada a maiores valores de dureza, gomosidade, adesividade e viscosidade, e estes foram obtidos nos doces sem adição de amido e com concentração de 0 e $15 \%$ de soro.

\section{AGRADECIMENTOS}

Os autores agradecem aos auxílios financeiros e as bolsas de produtividade da FAPEMIG, CNPq e CAPES.

\section{REFERÊNCIAS}

BRASIL. Ministério da Agricultura e do Abastecimento. Portaria $n^{0} 354$, de 04 de setembro de 1997. Regulamento Técnico para Fixação de Identidade e Qualidade de Doce de Leite. Diário Oficial da República Federativa do Brasil: seção 1, Brasília, DF, n. 172, p. 19.685, 08 set. 1997.

BRASIL. Ministério da Agricultura, Pecuária e Abastecimento. Secretaria de Defesa Agropecuária. Manual de Métodos Oficiais para Análise de Alimentos de Origem Animal. 1. ed. Brasília: MAPA, 2018. 140 p.

CARNEIRO, L. C. M. et al. Influência de diferentes reguladores de acidez na estabilização coloidal do leite e nas características físicoquímicas de doce de leite. Indústria de Laticínios, v. 142, p. 68-74, 2020.

F R A N C I S Q U IN I, J . D. et al. 5-Hydroxymethylfurfural formation and color change in lactose-hydrolyzed Dulce de leche. Journal of Dairy Research, v. 86, p. $477-482,2019$.

FRANCISQUINI, J. D. et al. Avaliação da intensidade da reação de Maillard, de atributos físico-químicos e análise de textura em doce de leite. Revista Ceres, v. 63, p. 589-592, 2016.

FRANCISQUINI, J. D. et al. Evolution of 
soluble solid content and evaporation rate curves during the manufacture of dulce de leche (dl). Ciência e Tecnologia de Alimentos, v. 39, p. 78-82, 2018a.

FRANCISQUINI, J. D. et al. Physicochemical and compositional analyses and 5-hydroxymethylfurfural concentration as indicators of thermal treatment intensity in experimental dulce de leche. Journal of Dairy Research, v. 85, p. 1-6, 2018 b.

HUIDOBRO, F. R. et al. A comparison between two methods (Warner-Bratzler and texture profile analysis) for testing either raw meat or cooked meat. Meat Science, n. 69, p. 527-536, 2005.

OliveirA, A. C. P. et al. Parâmetros industriais da produção de doce de leite. Revista do Instituto de Laticínios Cândido Tostes, v. 71, p. 179-185, 2017.

PAIVA, V. N. et al. Desafios tecnológicos na produção de produtos com baixo teor de lactose. Revista do Instituto de Laticínios Cândido Tostes, v. 73, p. 91-101, 2018.

PERRONE, I. T. et al. Atributos tecnológicos de controle para produção do doce de leite. Revista do Instituto de Laticínios Cândido Tostes, v.67, n. 385, p. 42-51, 2012.
PERRONE, I. T.; COSTA JÚNIOR, L. C. G.; MAGALHÃES, F. A. R. Características tecnológicas, de rendimento e cristalização em doce de leite obtido de mistura de leite e soro de fabricação de queijo. Revista do Instituto de Laticínios Cândido Tostes, v. 63, n. 361, p. 3-8, 2008.

PERRONE, I. T.; FERRUA, F. Q.; ABREU, L. R. Efeito da nucleação secundária sobre a cristalização do doce de leite. Revista do Instituto de Laticínios Cândido Tostes, v. 61, p. 1-4, 2006.

PERRONE, I. T.; STEPHANI, R.; NEVES, B. S. Doce de Leite: Aspectos Tecnológicos. Juiz de Fora: do autor, 2011. 186p.

SILVA, F. L et al. Efeito do soro de leite no processo de concentração do doce de leite pastoso. Revista do Instituto de Laticínios Cândido Tostes, v. 68, n. 394, p. 20-24, 2013.

SILVA, F. L. et al. Production of dulce de leche: The effect of starch addition. LWT Food Science and Technology, v. 62, p. $417-423,2015$.

SZCZESNIAK, A. S. Objective measurements of food texture. Journal of Food Science, v. 28, p. 410-420, 1963. 